\title{
Roles of circular RNAs in immune regulation and autoimmune diseases
}

\author{
Zheng Zhou', Bao Sun ${ }^{2,3}$, Shiqiong Huang ${ }^{2,3}$ and Lingling Zhao ${ }^{1}$
}

\begin{abstract}
Circular RNAs (circRNAs), as a novel class of endogenously expressed non-coding RNAs (ncRNAs), have a high stability and often present tissue-specific expression and evolutionary conservation. Emerging evidence has suggested that circRNAs play an essential role in complex human pathologies. Notably, circRNAs, important gene modulators in the immune system, are strongly associated with the occurrence and development of autoimmune diseases. Here, we focus on the roles of circRNAs in immune cells and immune regulation, highlighting their potential as biomarkers and biological functions in autoimmune diseases, such as systemic lupus erythematosus (SLE), rheumatoid arthritis (RA), multiple sclerosis (MS), primary biliary cholangitis (PBC), and psoriasis, aiming at providing new insights into the diagnosis and therapy of these diseases.
\end{abstract}

\section{Facts}

- CircRNAs are related to various biological processes in immune cells, as well as immune regulation under multifarious physiological and pathological conditions.

- CircRNAs serve as potential biomarkers for the diagnosis and severity of certain autoimmune diseases, such as systemic lupus erythematosus (SLE), rheumatoid arthritis (RA), multiple sclerosis (MS), primary biliary cholangitis (PBC).

- CircRNAs contribute to the development of autoimmune diseases by acting as miRNA sponges to regulate many biological processes, including DNA methylation, immune response, and inflammatory response.

- Certain circRNAs, such as cia-cGAS and dsRNA-

Correspondence: Lingling Zhao (zhaolingling6@163.com)

'Department of Chinese Medicine, The First Affiliated Hospital of Zhengzhou University, Zhengzhou 450000, China

²Department of Clinical Pharmacology, Xiangya Hospital, Central South University, Changsha 410000, China

Full list of author information is available at the end of the article.

These authors contributed equally: Zheng Zhou, Bao Sun

Edited by H.-U. Simon containing circRNAs, may act as potential targets for the treatment of autoimmune diseases.

\section{Open questions}

- What is the molecular mechanism by which circRNAs trigger autoimmune diseases?

- Are circRNAs effective and universal biomarkers for the diagnosis and severity of autoimmune diseases?

- How autoimmune diseases are linked to circRNAs' biogenesis, cytoplasmic accumulation and even posttranscriptional modifications?

- Is there potential for practical clinical applications based on findings concerning certain circRNAs?

\section{Introduction}

Normally, immune cells have receptors that can distinguish between self (ie, healthy native structures) and nonself or deviant self (ie, pathogens or tumor antigens), enabling these cells to discover pathogens or malignantly transformed cells. At the same time, precise regulation of certain immune-related genes is essential to an organism's ability to generate strong immunity to pathogens while limiting autoimmunity to self-antigens. Once immunodeficiency or immune dysregulation, people may suffer 
from immune system diseases, chronic infections, and even cancer. Typically, autoimmune diseases are a type of complex multifactorial diseases with characteristics of the presence of autoreactive immune cells and specific autoantibodies. According to statistics, there are $>100$ human diseases in the world that are known as autoimmune or chronic inflammatory, which are believed to affect $5-10 \%$ of individuals ${ }^{1}$.

Generally, circular RNAs (circRNAs) are widely studied non-coding RNA (ncRNA). Although RNA molecules are traditionally considered to be passive carriers of genetic information from DNA sequences to protein synthesis, lots of research has revealed that ncRNAs are critical participators in the process of gene expression ${ }^{2,3}$. To date, accumulated evidence has shown that circRNAs play an important role in various physiological and pathological processes, such as cancer ${ }^{4,5}$, cardiovascular diseases ${ }^{6}$, and neuronal diseases ${ }^{7}$. Intriguingly, circRNAs serve important functions in antiviral immunity ${ }^{8}$. Furthermore, circRNAs are aberrantly expressed in patients with systemic lupus erythematosus (SLE), some of which may serve as new non-invasive biomarkers for this autoimmune disease $^{9}$. Therefore, an in-depth study of circRNAs will not only increase our understanding of the molecular mechanisms that underlie autoimmune diseases, but also provide future potential treatment of these diseases. In this review, we emphasize the potential roles of circRNAs in certain autoimmune diseases, including SLE, rheumatoid arthritis (RA), multiple sclerosis (MS), primary biliary cholangitis (PBC), and psoriasis.

\section{Biogenesis and functions of circRNAs}

Unlike the characteristics of linear RNA molecules, circRNA has a special structure that is a covalently closed loop without $5^{\prime}$ end caps and 3' Poly (A) tails ${ }^{10,11}$. This RNA species was first identified in RNA viruses in $1976^{12}$ and subsequently discovered in the cytoplasm of eukaryotic cells ${ }^{13}$ and yeast mitochondria ${ }^{14}$. With the development of high-throughput sequencing technology and microarray technique, plenty of circRNAs have been successfully discovered in various organisms in nature. In most cases, circRNAs are produced by "back-splicing" events of the precursor messenger RNAs (pre-mRNAs), in which a downstream $5^{\prime}$ splice donor is linked to an upstream $3^{\prime}$ splice acceptor via a $3^{\prime} \rightarrow 5^{\prime}$ phosphodiester bond ${ }^{15,16}$. According to their components, circRNAs are mainly divided into three types: exonic circular RNAs (ecircRNAs) ${ }^{17}$, intronic circular RNAs $(\mathrm{ciRNAs})^{18}$, and exon-intron circular RNAs (EIciRNAs) ${ }^{19}$, among which ecircRNAs occupy the vast majority.

Previous studies have found that RNA polymerase II (Pol II) elongation rate is associated with the efficiency and results of splicing ${ }^{20,21}$. The fast Pol II elongation may facilitate reverse complementary sequences across long flanking introns to pair up for back-splicing, thereby promoting circRNA formation ${ }^{22}$. Several possible circRNA biogenetic pathways, including "complementary sequence-mediated circularization", "lariat-driven circularization" and "RNA-binding proteins-mediated circularization", have been proposed. Liang et al..$^{23}$ found that certain introns containing both splice sites and flanking inverted complementary repeats, such as Alu elements, were necessary for the circularization of the intervening exons in cells. In this process, the intronic repeat sequences must be base-paired with each other to bring the splice sites close together, thereby facilitating backsplicing. Notably, when a pre-mRNA has multiple intronic repeat sequences, the competitive pairing between the repeat sequences result in alternative circularization, thus affecting the splicing outcomes ${ }^{24}$. For example, this alternative circularization can cause a single gene to form multiple different circRNA transcripts ${ }^{25}$. Another form of circRNA generation is associated with exon skipping, in which a lariat precursor containing one or more skipped exons is first generated ${ }^{26,27}$. Then, the lariat removes its internal intron sequences to generate a mature circRNA and a double lariat. In some situations, the intervening introns in the encircled exons are not removed, which yields the so-called EIciRNA ${ }^{16}$. In addition, some RNAbinding proteins including the muscleblind, nuclear factor 90/nuclear factor 110 (NF90/NF110) and alternative splicing factor Quaking (QKI) were reported to promote back-splicing events by increasing the interaction between upstream and downstream introns ${ }^{28-30}$. CiRNAs are produced by intron lariats that fail to be degraded and debranched, and they do not contain linear $3^{\prime}$ tails ${ }^{18}$. To some extent, these models explain the molecular mechanisms of circRNA biosynthesis (Fig. 1).

Usually, circRNAs work as molecular sponges for microRNAs (miRNAs), thereby regulating miRNA target gene expression ${ }^{31,32}$. Hansen et al. ${ }^{31}$ first found that a highly expressed circRNA (ciRS-7) could be used as a miR-7 sponge in human and mouse brains. This circRNA contained $>70$ conserved binding sites for miR-7, and it was strongly inhibited miR-7 activity by binding to miR-7, resulting in elevated levels of miR-7 targets. Subsequently, increasing evidence showed that various circRNAs could adsorb miRNAs, thus participating in many physiological and pathological processes ${ }^{33,34}$. For example, Hsa_circ_0009361 could regulate the expression of adenomatous polyposis coli 2 by binding to miR-582, thereby inhibiting the progression of colorectal cancer ${ }^{35}$. Furthermore, a class of EIciRNAs localized in the nucleus promoted the transcription of their parental genes in cis through interacting with U1 snRNP, indicating that these circRNAs could regulate gene expression via specific RNA-RNA interplay between U1 snRNA and EIciR$\mathrm{NAs}^{19}$. Conn et al. ${ }^{36}$ demonstrated that a circRNA derived 


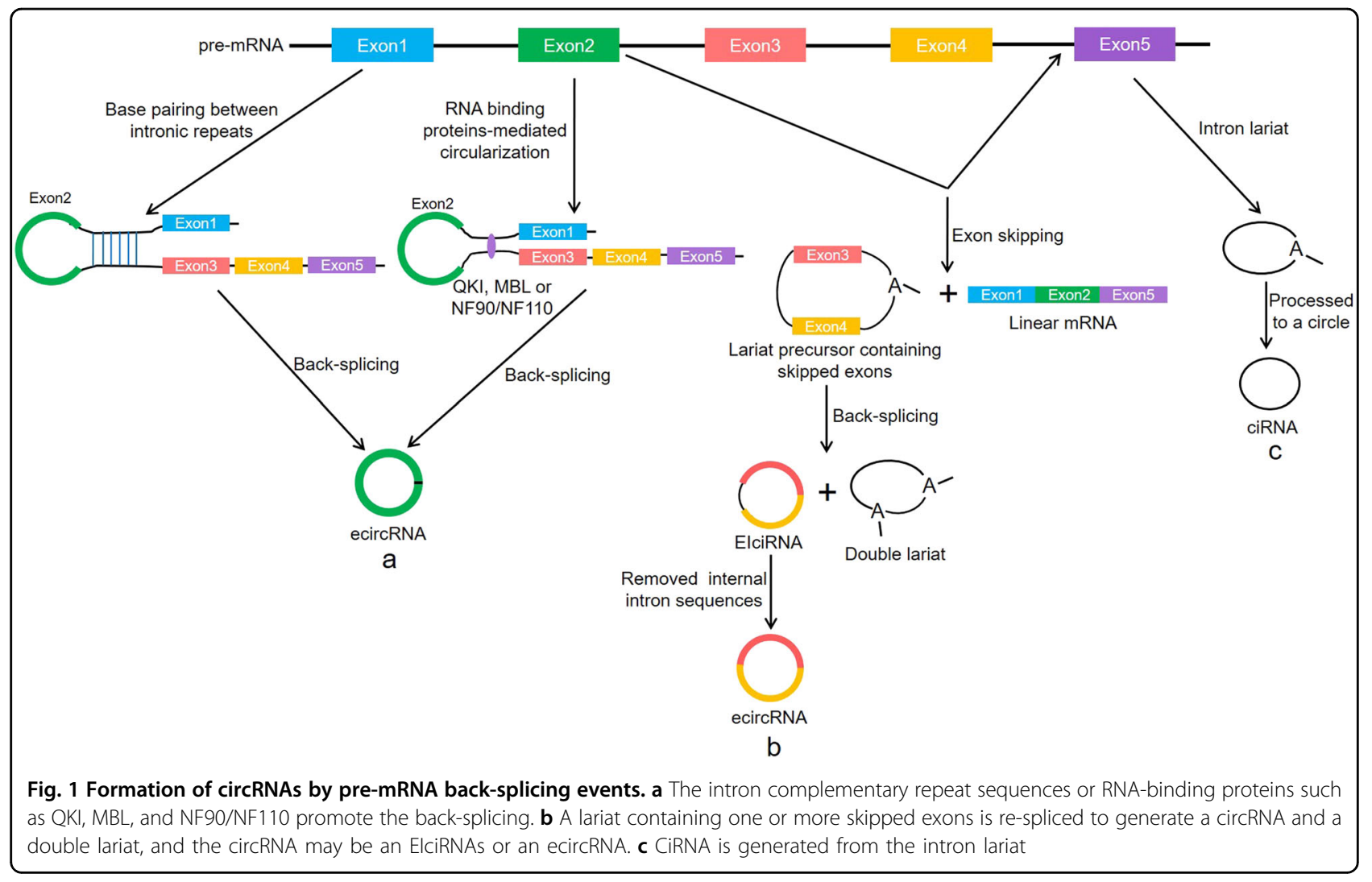

from the exon 6 of SEPALLATA3 gene bound to its cognate DNA locus to form an R-loop and thus regulated the splicing of its cognate mRNA. Notably, the circular form of long intergenic non-protein-coding RNA p53induced transcript could encode an 87 amino-acid regulatory peptide, which bound to polymerase associated factor complex (PAF1c) and inhibited the transcriptional elongation in glioblastoma ${ }^{37}$. These findings show that there may be more biological functions of circRNA than previously predicted.

\section{circRNAs in immunity}

\section{circRNAs in immune cells}

Indeed, some studies have demonstrated the diverse biological functions of circRNAs in immune cells. Hematopoietic stem cells (HSCs) can differentiate into a variety of progenitor cells, which subsequently generate all kinds of specialized blood cells, such as red blood cells, megakaryocytes, myeloid cells, and lymphocytes ${ }^{38}$. Nicolet et al. $^{39}$ found that circRNA showed cell-specific expression in human hematopoietic progenitors as well as differentiated lymphoid and myeloid cells. For example, during hematopoietic differentiation, the expression levels of circRNA of lymphocytes were the highest, and the high levels were reflected in abundance rather than variety. Moreover, circ-FNDC3B showed the highest expression level in natural killer cells, while circ-ELK4, circ-MYBL1, and circ-SLFN12L showed the highest expression in $\mathrm{T}$ cells and natural killer cells. Macrophages are an essential part of innate immunity and can be induced to diverse phenotypes under different external stimuli ${ }^{40}$. A recent study explored the expression of circRNAs in macrophages under two different polarization conditions (M1 macrophages induced by interferon- $\gamma$ (IFN- $\gamma$ ) and lipopolysaccharide (LPS), and M2 macrophages induced by interleukin-4 (IL-4) $)^{41}$. The results showed that 189 circRNAs were differentially expressed in the M1 compared with the M2 macrophages. To further elucidate the implication of the differentially expressed circRNAs, the researchers also predicted the miRNAs that interacted with them. For the overexpressed circRNA-010231 in $\mathrm{M} 1$, the five miRNA response elements with good scores were miR-1964-5p, miR-19b-2-5p, miR-141-5p, miR-6950-5p, and miR-145a-5p, respectively. These findings provide new ideas for the roles of circRNAs in the polarization of macrophages. Recently, Agirre et al. ${ }^{42}$ found that 1356 new identified circRNAs were expressed in human humoral immune response, as well as plasma cells (tonsillar plasma cells and bone marrow plasma cells) had the highest average expression levels. The expression of these circRNAs was significantly negatively correlated with the levels of some RNA-binding proteins 


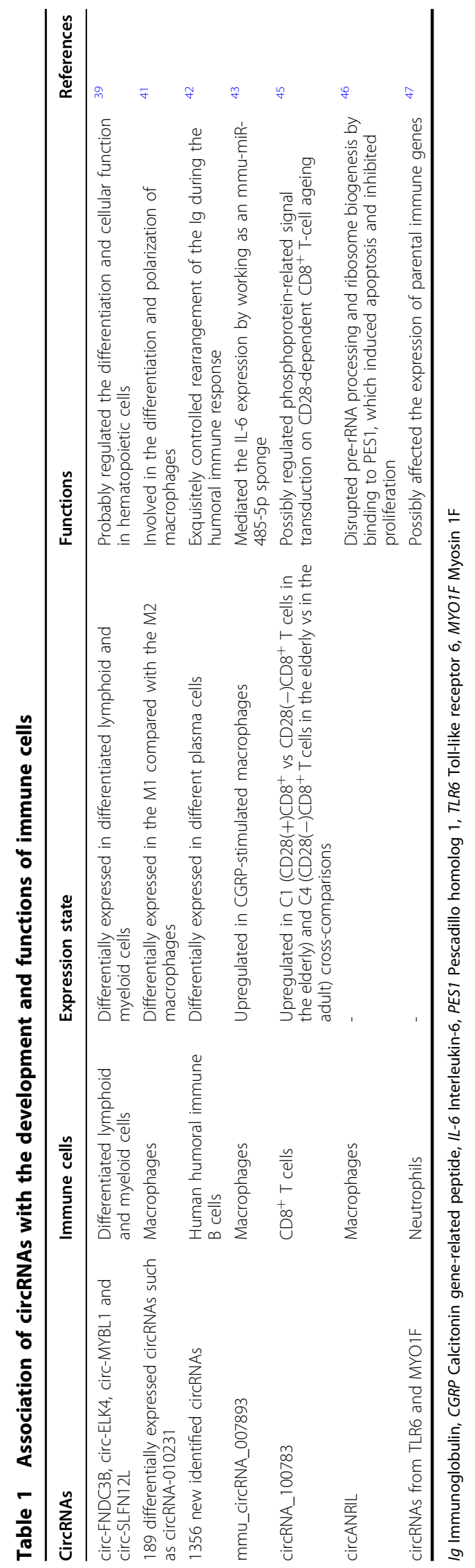

including adenosine deaminase acting on RNA 1, DEAH box helicase 9, and heterogeneous nuclear ribonucleoprotein $\mathrm{L}$, suggesting that these RNA-binding proteins might be involved in the biogenesis of circRNAs during terminal B-cell differentiation. Notably, the circRNAs in human plasma cells were mainly derived from immunoglobulin (Ig) genes and represented the combinatorial clonal state of Ig loci.

Calcitonin gene-related peptide (CGRP) could induce the expression of IL- 6 in macrophages ${ }^{43}$. In this process, mmu_circRNA_007893 mediated the IL-6 expression by working as an endogenous mmu-miR-485-5p sponge. When macrophages were stimulated by CGRP, mmu_circRNA_007893 was significantly increased. However, after silencing mmu_circRNA_007893, the level of mmumiR-485-5p was increased while IL-6 mRNA expression was decreased. During the process of immunosenescence, there was a significant feature that the proportion of CD8 $\mathrm{T}$ lymphocytes lacking CD28 expression would be increased $^{44}$. Wang et al. $^{45}$ discovered that circRNA_100783 in ageing human $\mathrm{CD}^{+} \mathrm{T}$ cells might function as a new biomarker for CD28-related $\mathrm{CD}^{+} \mathrm{T}$ cell ageing. By further investigating the circRNA_100783targeted miRNA-mRNA network, they observed that circRNA_100783 might be mainly related to alternative splicing events, the production of splice variants and the expression of phosphoprotein. Interestingly, circANRIL has been shown to disrupt pre-rRNA processing and ribosome biogenesis by binding to pescadillo homolog 1 in vascular smooth muscle cells and macrophages, leading to the nucleolar stress and activation of p53, which in turn induced apoptosis and inhibited proliferation ${ }^{46}$.

By measuring the expression profiles of circRNAs in 20 human tissues that were highly correlated with diseases, Maass et al. ${ }^{47}$ demonstrated that many circRNAs showed tissue-specific expression and could be closely related to the clinical phenotypes and mechanisms of human diseases. At the same time, they found that immune-related components, toll-like receptor 6 (TLR6), and myosin $1 \mathrm{~F}$ (MYO1F), could produce circRNAs in neutrophils, suggesting that the circRNAs were likely to be involved in neutrophil immune responses. In addition, $\mathrm{Li}$ et $\mathrm{al}^{48}$ found that a W chromosome-linked circRNA was female-biased expression in a kind of flatfish (halfsmooth tongue sole) and tended to be expressed in some immune tissues, especially head kidney and spleen. Importantly, the expression of this circRNA in spleen was significantly upregulated after infection, indicating that it might be related to the immune response. In summary, circRNAs actively participate in various biological processes in immune cells, such as differentiation, polarization, immune response, senescence, and apoptosis (Table 1). 


\section{circRNAs in immune regulation}

In recent years, increasing evidence linked circRNAs to immune regulation under multifarious physiological and pathological conditions, including anti-infection immunity $^{49,50}$, tumor immunity ${ }^{51,52}$, the activation of inflammation $^{53}$, and even organ transplantation ${ }^{54}$. Through next-generation sequencing technology, Ma et al. ${ }^{55}$ found that 123 circRNAs were differentially expressed in Mockand transmissible gastroenteritis virus (TGEV)-infected porcine intestinal epithelial cell line. Furthermore, Kyoto Encyclopedia of Genes and Genomes (KEGG) analysis suggested that the mRNAs in circRNA-miRNA-mRNA regulatory network were most significantly involved in inflammation and immune response, including retinoic acid-inducible gene-I (RIG-I)-like receptor, tumor necrosis factor (TNF), NOD-like receptor (NLR), TLR, and nuclear factor- $\kappa \mathrm{B}(\mathrm{NF}-\mathrm{kB})$ pathway. Of note, SsC_circRNA_009380 could promote the activation of NF$\kappa B$ pathway via interacting with miR-22, thereby mediating TGEV-induced inflammation. Analogously, another study analyzed the circRNA expression profiles and circRNA-associated competing endogenous RNA (ceRNA) network of early HIV infection (EHI) patients ${ }^{56}$. The results indicated that 1365 circRNAs were abnormally expressed in HARRT-naive EHI patients in contrast to healthy controls, and the targeting mRNAs among the ceRNA networks were mainly related to inflammatory response, immune response and defense response to virus infection. Actually, circRNAs were closely related to the immune factors NF90/NF110 in viral infection ${ }^{29}$. Specifically, NF90/NF110 enhanced pre-mRNA back-splicing by stabilizing the intron complementary sequence pairs in the nucleus and interacted with mature circRNAs to form complexes in the cytoplasm. After viral invasions, NF90/ NF110 in the nucleus were transported to the cytoplasm, and then the levels of circRNAs were reduced. At the same time, NF90/NF110 could be released from the complexes and subsequently suppressed viral replication by binding to viral mRNAs.

Meaningfully, Fu et al. ${ }^{57}$ found that 171 circRNAs were dysregulated in peripheral blood mononuclear cells (PBMCs) of patients with active tuberculosis (TB). Of these, circRNA_103017, circRNA_101128, and circRNA_059914 were expected to serve as new biomarkers for active TB. What's more, circRNA_101128 could contribute to the pathogenesis of $\mathrm{TB}$ by regulating miRNA let-7a. In LPS-stimulated mouse macrophages, $\mathrm{Ng}$ et al. $^{58}$ observed that one circRNA, mcircRasGEF1B, was regulated by TLR4 pathway. Moreover, the knockdown of mcircRasGEF1B reduced the expression of mature intercellular adhesion molecule-1 (ICAM-1) via modulating the stability of ICAM-1 mRNAs. Noteworthily, ICAM-1 was related to the pulmonary neutrophil recruitment in LPS-induced airway disease ${ }^{59}$, and could also suppress the polarization of M2 macrophages through the blockade of efferocytosis in tumor microenvironment ${ }^{60}$, implicating its various roles in innate immune response.

A recent study showed that hsa_circ_0005105 could facilitate the expression of inflammatory cytokines by regulating the miR-26a-targeted nicotinamide phosphoribosyltransferase, which provided a new target for the treatment of osteoarthritis $(\mathrm{OA})^{61}$. In contrast to nonlesional skin of severe acne patients, Liang et al. ${ }^{62}$ discovered that up to 538 circRNAs were aberrantly expressed in adjacent lesional skin, and these circRNAs were mainly connected with the biological pathways such as inflammation, metabolism, and immune response. In addition, has_circ_0020397 could promote the expression of telomerase reverse transcriptase and programmed death-ligand 1 (PD-L1) by binding to miR-138, thereby regulating the viability, apoptosis and invasion of colorectal cancer cells ${ }^{63}$. Studies have shown that PD-L1 is closely related to tumor escape from immune control ${ }^{64,65}$, so has_circ_0020397 may promote tumor development by regulating tumor immunity. These findings demonstrate that circRNAs are crucial participants in immune regulation (Fig. 2). Hence, it is foreseeable that dysregulation of these functions is very likely to be involved in the development of autoimmune diseases.

\section{circRNAs in autoimmune diseases}

Autoimmune diseases, mainly characterized by a damaged immune system and the loss of immune tolerance to self-antigens, are a group of heterogeneous conditions ${ }^{66}$. Although the molecular mechanisms are still largely unknown, increasing evidence indicates that the complex interplay of environmental factors and epigenetic dysregulation facilitate the pathogenesis of these diseases in genetically susceptible individuals ${ }^{67,68}$. As described above, circRNAs are closely associated with the immune system. Meanwhile, recent studies have demonstrated that circRNAs are not only involved in the pathogenesis of autoimmune diseases, but also represent non-invasive biomarkers for them (Fig. 3).

\section{circRNAs in SLE}

SLE is a chronic autoimmune disease that predominately affects women of childbearing age. Its main features are the autoreactive $\mathrm{B}$ and $\mathrm{T}$ lymphocytes as well as the overproduction of antibodies targeting self-antigens ${ }^{69}$. Unfortunately, SLE can result in multi-organ pathologies and a wide range of clinical manifestations, including arthritis, central nervous system disease, renal disease and skin disease ${ }^{70}$. Although SLE is immune-mediated, the pathogenic mechanisms are still not fully understood. 


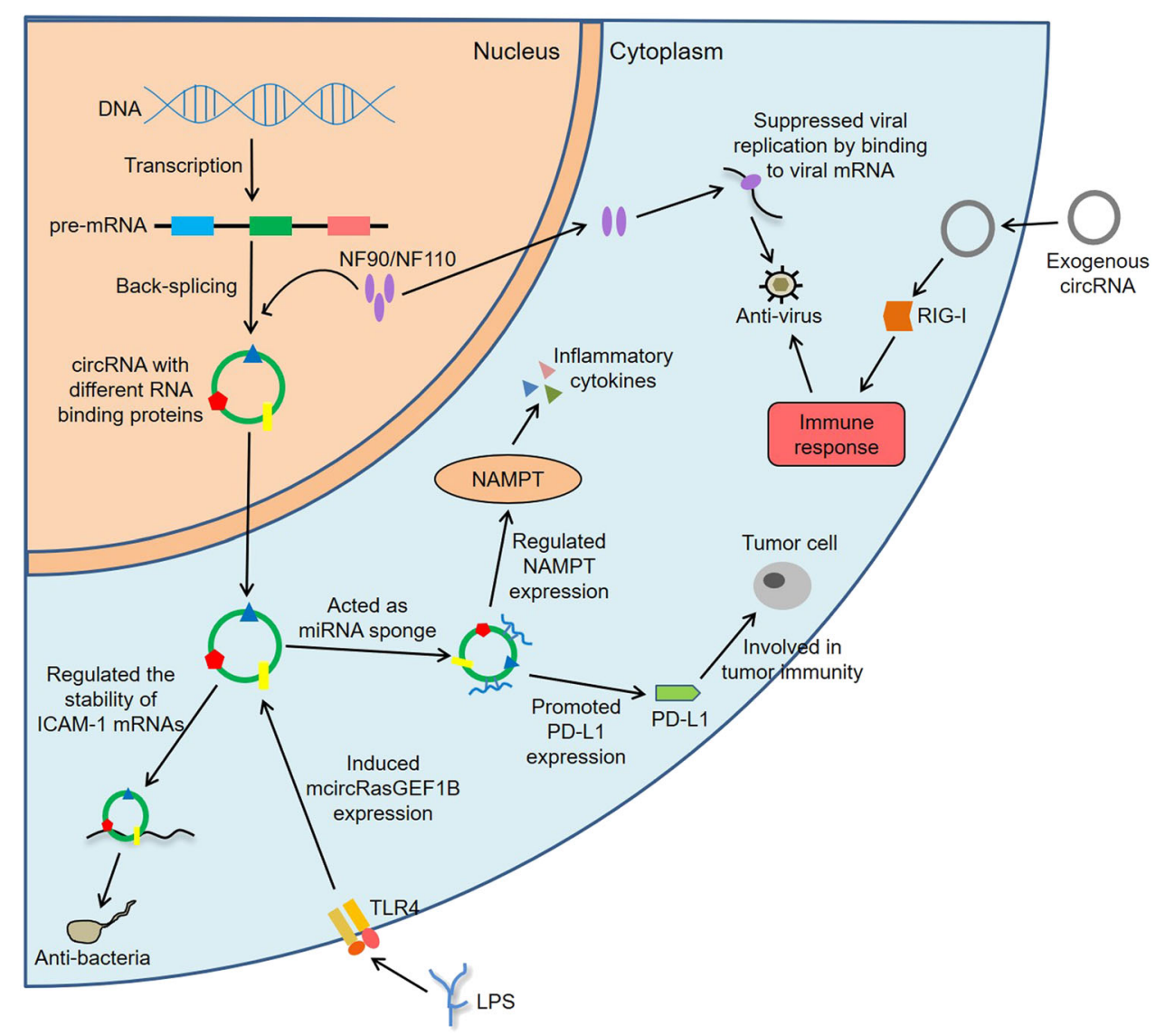

Fig. 2 CircRNAs in immune regulation. NF90/NF110 enhance back-splicing by stabilizing the intron complementary sequence pairs in the nucleus and are exported to the cytoplasm to suppress viral replication after viral infection. The exogenous circRNA induces innate immune response by activating RIG-I, whereas the endogenous circRNA binds to different RNA-binding proteins that reflect its endogenous biogenesis. hsa_circ_0005105 facilitates the expression of inflammatory cytokines by regulating the miR-26a targeted NAMPT. has_circ_0020397 promotes the expression of PD-L1 by binding to miR-138, thereby participating in tumor immunity. In addition, mcircRasGEF1B induced by LPS is involved in anti-bacteria immunity by modulating the stability of ICAM-1 mRNAs

\section{T-cell circRNAs in SLE}

Recently, 127 differentially expressed circRNAs were identified in T cells of SLE patients, and a downregulated circRNA, hsa_circ_0045272, was further verified by quantitative $\mathrm{PCR}^{71}$. Mechanism research revealed that the knockdown of this circRNA significantly upregulated the early apoptosis and enhanced the production of IL-2 in activated Jurkat cells. Zhang et al. $^{72}$ supported that hsa_circ_0012919 was aberrantly upregulated in CD4 $4^{+}$ $T$ cells of SLE patients. Meanwhile, the downregulation of hsa_circ_0012919 increased the expression of DNA methyltransferase 1 (DNMT1), whereas reduced the expression of CD70 and CD11a in $\mathrm{CD}^{+} \mathrm{T}$ cells from inactive and active SLE patients. The inhibition of hsa_circ_0012919 also rescued the DNA hypomethylation of CD70 and CD11a in CD4 ${ }^{+} \mathrm{T}$ cells of SLE patients, which could be reversed by downregulation of DNMT1. Strikingly, this circRNA could regulate the expression of regulated on activation, normal $\mathrm{T}$ cell expressed and secreted (RANTES) and Kruppel-like factor 13 (KLF13) by bonding to miR-125a-3p. It has been confirmed that migration rate of basophils to RANTES and monocyte chemotactic protein 1 (MCP-1) is remarkably increased in SLE patients, which is possibly associated with tissue damage in $\mathrm{SLE}^{73}$. KLF13 could positively regulate RANTES and was related to the expression of IL-4 in $\mathrm{CD}^{+}{ }^{+} \mathrm{T}$ cells ${ }^{74}$.

\section{PBMC circRNAs in SLE}

Wang et al. $^{75}$ found downregulation of circIBTK and upregulation of miR-29b in PBMCs of SLE patients, both of which were correlated with anti-double-stranded DNA, SLE Disease Activity Index (SLEDAI) score and complement component 3 (C3) level. Importantly, circIBTK could inhibit the DNA demethylation and activation of protein kinase B (AKT) by binding to miR-29b in SLE. Many studies have shown that AKT signaling pathway can regulate the functions of immune cells, and its 


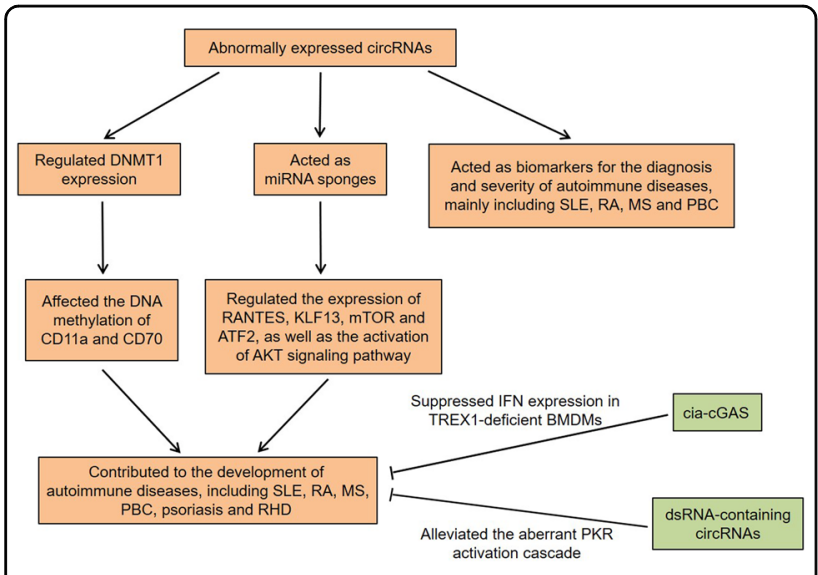

Fig. 3 Roles of circRNAs in autoimmune diseases. CircRNAs contribute to the development of autoimmune diseases by regulating various biological processes, such as DNA methylation, immune response, and inflammatory response. Furthermore, circRNAs may be used as potential biomarkers for the diagnosis and severity of autoimmune diseases. The overexpression of cia-cGAS can suppress IFN expression in TREX1-deficient BMDMs. The overexpression of dsRNA-containing circRNAs alleviate the aberrant PKR activation cascade in SLE patient-derived cells

dysregulation leads to the progression of SLE. For instance, AKT could coordinate IL-2 signaling and T-cell antigen receptor to hold the expression of adhesion molecules, cytolytic effector molecules, as well as cytokine and chemokine receptors in cytotoxic $\mathrm{T}$ cells ${ }^{76}$. Another research showed that hsa_circ_0049224 and has_circ_0049220 were underexpressed in PBMCs of inactive and active SLE patients ${ }^{77}$. Moreover, the levels of these two circRNAs were negatively correlated with SLEDAI and the degree of SLE severity, which indicated that they might be regarded as markers for the activity and severity of SLE.

\section{Plasma circRNAs in SLE}

In addition, hsa_circ_400011, hsa_circ_102584, hsa_circ_101471, and hsa_circ_100226 were abnormally expressed in plasma of SLE patients ${ }^{9}$. Through bioinformatics analysis, the researchers discovered multiple MREs of hsa_circ_100226, including hsa-miR-24-3p, hsa-miR875-3p, hsa-miR-138-5p, hsa-miR-620, and hsa-miR-145$3 p$. Among them, decreased miR-138 could enhance NF$\kappa B$ activation via suppressing the expression of $p 65$ in the chondrocytes, triggering the inflammatory response ${ }^{78}$. Moreover, miR-138-5p regulated extracellular matrix catabolism and inflammation, thereby affecting the progression of $\mathrm{OA}^{79}$. Interestingly, the upregulated circRNA_002453 level in plasma of lupus nephritis (LN) patients was related to the severity of renal involvement ${ }^{80}$. Although the level of circRNA_002453 had no significant correlation with disease activity, it was positively associated with renal SLEDAI score and 24-hour proteinuria.

\section{circRNAs in RA}

RA is a prevalent autoimmune disorder characterized by generalized inflammation in multiple joints, which always results in serious cartilage and bone erosion as well as articular deformation ${ }^{81}$. Rheumatoid factor (RF), anticarbamylated protein (anti-CarP), and anti-cyclic citrullinated peptide-2 (anti-CCP2) are the most well-known autoantibodies in this disease ${ }^{82}$.

\section{PBMC circRNAs in RA}

Latest studies revealed that the levels of hsa_circ_0058794 and hsa_circ_0092285 were markedly increased in PBMCs of patients with RA, and the levels of hsa_circ_0038644 and hsa_circ_0088088 were decreased $^{83}$. Actually, hsa_circ_0038644 was spliced from the protein kinase $\mathrm{C}$ beta gene, which was related to the activation of NF- $\mathrm{kB}^{84}$. Furthermore, the expression of ciRS-7 was significantly upregulated in RA patients, and it could reduce the inhibitory effect of miR-7 on mammalian target of rapamycin (mTOR) by inhibiting the function of $\mathrm{miR}-7^{85}$. As the phosphatidylinositol-3-kinase/ AKT/mTOR (PI3K/AKT/mTOR) signaling pathway played an important role in synoviocyte proliferation and inflammatory responses ${ }^{86,87}$, ciRS-7 might be involved in the development of RA by regulating mTOR.

\section{Other cell type circRNAs in RA}

hsa_circ_0001859 was one of the differentially expressed circRNAs in synovial tissues of RA patients ${ }^{88}$. Mechanism studies found that this circRNA could promote activating transcription factors 2 expression and increase inflammatory activity by targeting miR-204/211. Furthermore, nuclear factor E2-related factor 2 (Nrf2), a potential therapeutic target for rheumatic diseases, could regulate many biological processes such as inflammation, immune response and cartilage and bone metabolism in the body ${ }^{89}$. By analyzing the circRNA expression profiles in the substantia nigra and corpus striatum of Nrf2knockout mice, Yang et al. $^{90}$ found that $\mathrm{mmu}_{-}$circRNA_34132, mmu-circRNA-015216 and mmu_circRNA_017077 were involved in the Nrf2-mediated neuroprotection against oxidative stress. Notably, the authors also uncovered that four mRNAs, Atp6v0a1, Atp6v0b, Atp6v0c, and Atp6v0e2, were enriched in RA pathway in the circRNA-miRNA-mRNA interaction network. Atp6v0c and Atp6v0e2 were potentially regulated by mmu_circRNA_017077 via binding to mmu-miR-346$3 \mathrm{p}$, and Atp6v0e2 and Atp6v0a1 were potentially regulated by mmu_circRNA_34132 via binding to $\mathrm{mmu}$-miR346-3p as well. These results supported that $\mathrm{mmu}_{-}-$ circRNA_34132 and mmu_circRNA_017077 might 
participate in the Nrf2-mediated development of RA by serving as molecular sponges for mmu-miR-346-3p.

\section{circRNAs in MS}

MS is a chronic disease of the central nervous system (CNS), and diffuse immune mechanisms as well as neurodegeneration are the underlying pathological processes in this disease. The peripheral immune response targeting the CNS occurs mainly in the early stage of MS, whereas immune process within the CNS dominates the progressive stage ${ }^{91}$. Most patients will develop permanent disability during the course of their disease, creating a huge burden for individual, family and society levels ${ }^{92}$.

Through the further characterization of Gasdermin B alternative splicing and back-splicing profiles, Cardamone et al $^{93}$ found that alternative splicing isoforms and an identified ecircRNA, containing exons 4 and 5, were significantly dysregulated in PBMCs of relapsing-remitting MS patients, which suggested that the abnormal RNA metabolism was involved in the pathogenesis of this disease. Metastasis associated lung adenocarcinoma transcript 1 (MALAT1) was an long non-coding RNA (lncRNA) that could regulate alternative splicing and has been shown to be associated with $\mathrm{MS}^{94}$. A systematic study found that the level of MALAT1 was upregulated in MS patients ${ }^{95}$. Meanwhile, 1114 alternative splicing events were significantly modulated and 49 circRNAs were differentially expressed in MALAT1-knockdown Jurkat T cells, a relevant cellular model for MS. Besides, the RNA-binding protein motif analysis showed a particular enrichment for the QKI in the exons modulated by MALAT1. Remarkably, QKI has been reported to regulate the formation of circRNAs ${ }^{30}$. These data indicate that MALAT1 dysregulation may lead to the development of MS by affecting splicing and back-splicing events.

\section{circRNAs in other autoimmune diseases}

Psoriasis is an inflammatory disease that mainly affects the skin and joints, and its pathophysiological characteristics are abnormal proliferation of keratinocytes and infiltration of immune cells in the dermis and epidermis ${ }^{96}$. Recently, Liu et al. ${ }^{97}$ discovered six downregulated and 123 upregulated circRNAs in skin mesenchymal stem cells (S-MSCs) of psoriatic lesions. Pathway analysis observed that the significantly downregulated mRNAs in the lesions mainly enriched in Janus kinase-signal transducer and activator of transcription (JAK-STAT) signaling, which was reported to participate in immune regulation ${ }^{98}$. Of these, a circRNA chr2:206992521| 206994966 could affect the activity of T lymphocytes in local lesions by regulating the secretion of certain cytokines, including IL-6, IL-11, and hepatocyte growth factor $^{97}$. In addition, hsa_circ_0061012, hsa_circ_0003689, chr4:121675708|121732604, and hsa_circ_0003718 were abnormally expressed in psoriatic lesions and might promote disease progression by interacting with miRNAs associated with psoriasis ${ }^{99,100}$.

$\mathrm{PBC}$ is a cholestatic, autoimmune-mediated liver disease that slowly progresses to portal fibrosis and biliary cirrhosis ${ }^{101}$. By carrying out the circRNA expression profiles, Zheng et al. ${ }^{102}$ found 22 aberrantly expressed circRNAs in plasma of $\mathrm{PBC}$ patients. It was worth noting that the level of hsa_circ_402458 was higher in PBC patients not treated with ursodeoxycholic acid (UDCA) than in those treated with UDCA. At the same time, the authors showed that hsa_circ_402458 might target two miRNAs, hsa-miR-943, and hsa-miR-522-3p. For miR-522-3p, it might be an effective target for regulating chronic inflammatory dis$\operatorname{order}^{103}$. Therefore, it can be speculated that hsa_circ_402458 may function as a miRNA sponge to regulate inflammation-related signaling pathways, thus contributing to the development of PBC.

In addition, by studying the circRNA expression profiles in atrial tissues from patients with persistent atrial fibrillation (AF) with rheumatic heart disease, $\mathrm{Hu}$ et al. ${ }^{104}$ predicted the potential roles of the differentially expressed circRNAs. The results suggested that 51 circRNAs were upregulated, and 57 circRNAs were downregulated in AF tissues compared with controls, respectively. Gene Ontology (GO) analysis revealed that the most significantly enriched biological process term was muscle contraction, the most significantly enriched cellular component term was muscle myosin complex, and the most significantly enriched molecular function term was muscle alpha-actinin binding. Meanwhile, KEGG pathway analysis indicated that the main involved pathways were dilated cardiomyopathy and hypertrophic cardiomyopathy.

circRNAs as potential biomarkers in autoimmune diseases

Owing to their stability, abundance, and evolutionary conservation, as well as their differential expression in patients with autoimmune diseases, circRNAs are likely to be potential biomarkers for these diseases. circPTPN22 derived from protein tyrosine phosphatase nonreceptor type 22 (PTPN22) was downregulated in the PBMCs of patients with $\mathrm{SLE}^{105}$. Importantly, the receiver operating characteristic (ROC) curve analysis showed that circPTPN22 had good diagnostic value for SLE. The downregulation of circPTPN22 was strongly negatively correlated with the SLEDAI scores, suggesting that this circRNA might be a biomarker for SLE diagnosis and disease severity. Zhang et al. ${ }^{106}$ observed that hsa_circRNA_407176 and hsa_circRNA_001308 were downregulated in both $\mathrm{PBMCs}$ and plasma of patients with SLE. Also, these two circRNAs in plasma and PBMCs might be candidate biomarkers for SLE, and their combination could improve the diagnostic efficiency. Even more, the level of hsa_circRNA_001308 was associated 
with $\mathrm{C}$ reactive protein and anti-sjögren's syndromerelated antigen $\mathrm{A}$ in plasma, as well as leukopenia in PBMCs. Analogously, hsa_circ_0003090 and hsa_circ_0057762 in whole blood could differentiate the patients with SLE from the healthy controls, indicating that these two circRNAs might have potential value for SLE diagnosis ${ }^{107}$.

By the analysis of ROC curve, Ouyang et al. ${ }^{108}$ found that circRNA_104871 in PBMCs was a strong predictor for RA. Likewise, another study found that hsa_circ_0044235 was significantly decreased in peripheral blood of patients with $\mathrm{RA}^{109}$. Meaningfully, according to the risk score based on hsa_circ_0044235, the researchers could effectively distinguish the patients with RA from those with SLE. Iparraguirre et al. ${ }^{110}$ indicated that circ_0035560 and circ_0005402 were underexpressed in peripheral blood leukocytes of MS patients and might function as dependable biomarkers for this disease. Interestingly, these two circRNAs were derived from annexin A2 (ANXA2), whose linear form was also downregulated in MS patients. Increasing evidence has shown that ANXA2 is involved in many autoimmune diseases, including antiphospholipid syndrome and LN, suggesting that circ_0035560 and circ_0005402 may be associated with the development of $\mathrm{MS}^{111,112}$. In summary, these studies provide a theoretical basis for the clinical application of circRNAs in autoimmune diseases.

\section{Conclusion and future perspectives}

Indeed, increasing evidence has identified that circRNAs are active participants in multiple stages of immune-cell development and immune regulation. Furthermore, circRNAs may not only be diagnostic biomarkers for human autoimmune diseases, but also represent the disease activity or severity. More importantly, circRNAs contribute to the development of autoimmune diseases by acting as miRNA sponges to regulate many biological processes, including DNA methylation, immune response, and inflammatory response (Table 2). Therefore, elucidating the roles of circRNAs in the setting of autoimmune disease will be a promising field.

Recently, Chen et al. ${ }^{113}$ found that the purified exogenous circRNA could induce innate immune response and confer a protective effect on viral infection by activating RIG-I. Further exploration indicated that cells could distinguish between self-nonself circRNAs based on the introns that produced them, and the reason might be that mature human circRNAs always bound to different RNA-binding proteins that reflect their endogenous splicing and biogenesis. In this regard, we speculate that the abnormal circRNAs in vivo, like foreign circRNAs, are involved in autoimmune diseases by activating the immune signaling. Interestingly, Xia et al. ${ }^{114}$ demonstrated that a novel circRNA, cia-cGAS, could protect dormant long-term-hematopoietic stem cells from cyclic GMP-AMP synthase (cGAS)-mediated exhaustion by suppressing the enzymatic activity of cGAS under homeostatic conditions. Meanwhile, the binding affinity of cia-cGAS for cGAS was stronger than that of self-DNA, thereby inhibiting cGAS-mediated generation of type I IFNs to maintain dormant HSCs. Three-prime repair exonuclease 1 (TREX1) was a major $3^{\prime} \rightarrow 5^{\prime}$ DNA exonuclease, whose dysregulation has been associated with some autoimmune diseases ${ }^{115,116}$. Conspicuously, the overexpression of cia-cGAS could suppress IFN expression in TREX1-deficient bone marrow derived macrophages (BMDMs), indicating that cia-cGAS could restrain autoimmune signaling in TREX1-deficient cells $^{114}$. Thus, cia-cGAS might act as a potential target for the treatment of autoimmune diseases by antagonizing cGAS. Recently, Liu et al. ${ }^{117}$ found that endogenous circRNAs tended to form 16-26 bp intra-molecular RNA duplexes and inhibited double-stranded RNA (dsRNA)activated protein kinase (PKR) activity by preferentially binding to PKR. The activity of RNase $\mathrm{L}$ in PBMCs derived from SLE patients was enhanced, accompanied by reduced circRNA expression and augmented PKR phosphorylation. Importantly, overexpression of dsRNAcontaining circRNAs could strongly attenuate the aberrant PKR activation cascade in SLE patient-derived cells, suggesting that circRNAs might serve as potential targets for the treatment of autoimmune diseases.

However, several important questions merit further resolution. To date, we have detailed the close relationship between autoimmune diseases and circRNAs, but little is known about the molecular mechanisms that trigger the pathogenesis. CircRNAs that act as endogenous miRNA sponges have been studied widely, but few circRNA/miRNA interactions have been experimentally validated in immunity ${ }^{61,63,118}$. It is suggested that exploring other mechanisms may improve the functional description of circRNAs in immunological contexts. Furthermore, circRNAs can exert potential biomarkers for various autoimmune diseases. Nonetheless, in many studies, the sample size of patients is relatively small, or the sample source has some limitations, which may affect the validity and universality of the conclusions that circRNAs can serve as biomarkers. Moreover, in-depth studies of circRNAs' biogenesis, accumulation in the cytoplasm, and even post-transcriptional modifications may increase our understanding of their biological functions.

In conclusion, continued investigation into circRNAs may yield more discoveries in the pathogenesis of autoimmune diseases and broaden the spectra of diagnosis and therapy for these diseases in the future. 


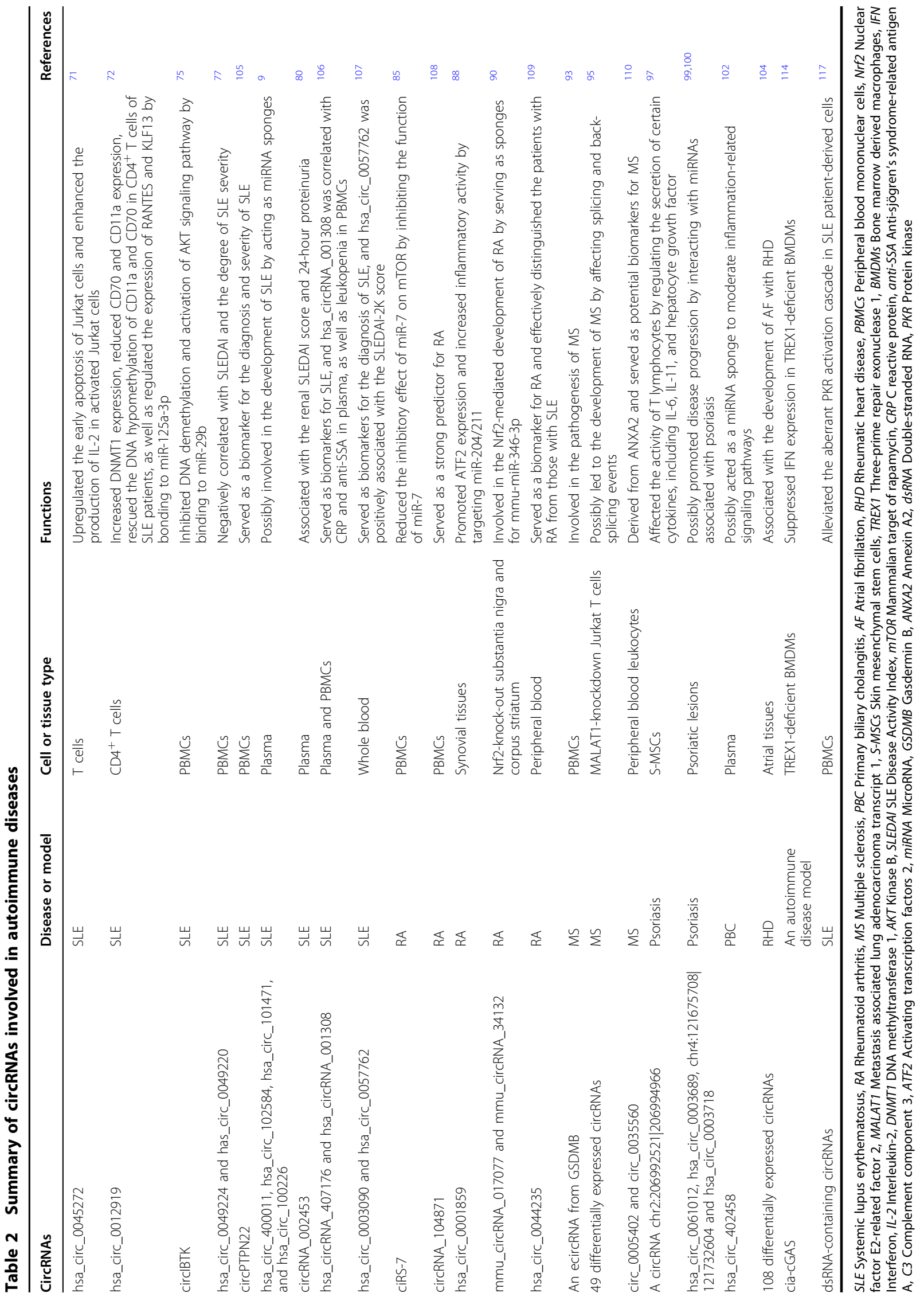




\section{Acknowledgements}

This research did not receive any specific grant from funding agencies in the public, commercial, or not-for-profit sectors.

\section{Author details}

'Department of Chinese Medicine, The First Affiliated Hospital of Zhengzhou University, Zhengzhou 450000, China. ${ }^{2}$ Department of Clinical Pharmacology, Xiangya Hospital, Central South University, Changsha 410000, China. ${ }^{3}$ Hunan Key Laboratory of Pharmacogenetics, Institute of Clinical Pharmacology, Central South University, Changsha 410000, China

\section{Conflict of interest}

The authors declare that they have no conflict of interest.

\section{Publisher's note}

Springer Nature remains neutral with regard to jurisdictional claims in published maps and institutional affiliations.

Received: 5 May 2019 Revised: 8 June 2019 Accepted: 12 June 2019 Published online: 26 June 2019

\section{References}

1. Shoenfeld, Y., Selmi, C., Zimlichman, E. \& Gershwin, M. E. The autoimmunologist: geoepidemiology, a new center of gravity, and prime time for autoimmunity. J. Autoimmun. 31, 325-330 (2008).

2. Marques-Rocha, J. L. et al. Noncoding RNAs, cytokines, and inflammationrelated diseases. FASEB J. y. 29, 3595-3611 (2015)

3. Zhou, Z., Sun, B., Huang, S., Jia, W. \& Yu, D. The tRNA-associated dysregulation in diabetes mellitus. Metabolism 94, 9-17 (2019).

4. Wang, K., Sun, Y., Tao, W., Fei, X. \& Chang, C. Androgen receptor (AR) promotes clear cell renal cell carcinoma (ccRCC) migration and invasion via altering the circHIAT1/miR-195-5p/29a-3p/29c-3p/CDC42 signals. Cancer Lett. 394, 1-12 (2017)

5. Guarnerio, J. et al. Oncogenic role of fusion-circRNAs derived from cancerassociated chromosomal translocations. Cell 165, 289-302 (2016).

6. Mehta, S. L., Pandi, G. \& Vemuganti, R. Circular RNA expression profiles alter significantly in mouse brain after transient focal ischemia. Stroke $\mathbf{4 8}$ 2541-2548 (2017)

7. Kumar, L. et al. Functional characterization of novel circular RNA molecule, circzip-2 and its synthesizing gene zip-2 in C. elegans model of parkinson's disease. Mol. Neurobiol. 55, 6914-6926 (2018).

8. Wang, M. et al. Circular RNAs: A novel type of non-coding RNA and their potential implications in antiviral immunity. Int. J. Biol. Sci. 13, 1497-1506 (2017).

9. $\mathrm{Li}, \mathrm{H}$. et al. Comprehensive circular RNA profiles in plasma reveals that circular RNAs can be used as novel biomarkers for systemic lupus erythematosus. Clin. Chim. Acta 480, 17-25 (2018).

10. Salzman, J., Gawad, C., Wang, P. L., Lacayo, N. \& Brown, P. O. Circular RNAs are the predominant transcript isoform from hundreds of human genes in diverse cell types. PLOS ONE 7, e30733 (2012).

11. Vicens, Q. \& Westhof, E. Biogenesis of circular RNAs. Cell 159, 13-14 (2014).

12. Sanger, H. L., Klotz, G., Riesner, D., Gross, H. J. \& Kleinschmidt, A. K. Viroids are single-stranded covalently closed circular RNA molecules existing as highly base-paired rod-like structures. Proc. Natl. Acad. Sci. USA 73, 3852-3856 (1976).

13. Hsu, M. T. \& Coca-Prados, M. Electron microscopic evidence for the circular form of RNA in the cytoplasm of eukaryotic cells. Nature 280, 339-340 (1979).

14 Arnberg, A. C., Van Ommen, G. J., Grivell, L. A., Van Bruggen, E. F. \& Borst, P. Some yeast mitochondrial RNAs are circular. Cell 19, 313-319 (1980).

15. Chen, L. L. \& Yang, L. Regulation of circRNA biogenesis. RNA Biol. 12, 381-388 (2015)

16. Holdt, L. M., Kohlmaier, A. \& Teupser, D. Molecular roles and function of circular RNAs in eukaryotic cells. Cell Mol. Life Sci. 75, 1071-1098 (2018).

17. Chen, I., Chen, C. Y. \& Chuang, T. J. Biogenesis, identification, and function of exonic circular RNAs. Wiley Interdiscip. Rev. RNA 6, 563-579 (2015).

18. Zhang, Y. et al. Circular intronic long noncoding RNAs. Mol. Cell 51, 792-806 (2013).
19. Li, Z. et al. Exon-intron circular RNAs regulate transcription in the nucleus. Nat. Struct. Mol. Biol. 22, 256-264 (2015).

20. Ip, J. Y. et al. Global impact of RNA polymerase II elongation inhibition on alternative splicing regulation. Genome Res. 21, 390-401 (2011).

21. Bentley, D. L. Coupling mRNA processing with transcription in time and space. Nat. Rev. Genet. 15, 163-175 (2014).

22. Zhang, Y. et al. The biogenesis of nascent circular RNAs. Ces Dev. 15, 611-624 (2016).

23. Liang, D. \& Wilusz, J. E. Short intronic repeat sequences facilitate circular RNA production. Genes Dev. 28, 2233-2247 (2014).

24. Zhang, X. O. et al. Complementary sequence-mediated exon circularization Cell 159, 134-147 (2014)

25. Ye, C. Y. et al. Full-length sequence assembly reveals circular RNAs with diverse non-GT/AG splicing signals in rice. RNA Biol. 14, 1055-1063 (2017).

26. Barrett, S. P., Wang, P. L. \& Salzman, J. Circular RNA biogenesis can proceed through an exon-containing lariat precursor. elife 4, e07540 (2015).

27. Zaphiropoulos, P. G. Circular RNAs from transcripts of the rat cytochrome P450 2C24 gene: correlation with exon skipping. Proc. Natl. Acad. Sci. USA 93, 6536-6541 (1996).

28. Ashwal-Fluss, R. et al. circRNA biogenesis competes with pre-mRNA splicing. Mol. Cell 56, 55-66 (2014).

29. Li, X. et al. Coordinated circRNA Biogenesis and Function with NF90/NF110 in Viral Infection. Mol. Cell 67, 214-227.e217 (2017).

30. Conn, S. J. et al. The RNA binding protein quaking regulates formation of circRNAs. Cell 160, 1125-1134 (2015).

31. Hansen, T. B. et al. Natural RNA circles function as efficient microRNA sponges. Nature 495, 384-388 (2013).

32. Memczak, S. et al. Circular RNAs are a large class of animal RNAs with regulatory potency. Nature 495, 333-338 (2013).

33. He, Q. et al. MOV10 binding circ-DICER1 regulates the angiogenesis of glioma via miR-103a-3p/miR-382-5p mediated ZIC4 expression change. J. Exp. Clin. Cancer Res. 38, 9 (2019).

34. Wu, K. et al. Circular RNA F-circSR derived from SLC34A2-ROS1 fusion gene promotes cell migration in non-small cell lung cancer. Mol. Cancer 18, 98 (2019).

35. Geng, Y. et al. Hsa_circ_0009361 acts as the sponge of miR-582 to suppress colorectal cancer progression by regulating APC2 expression. Clin. Sci. 133 1197-1213 (2019)

36. Conn, V. M. et al. A circRNA from SEPALLATA3 regulates splicing of its cognate mRNA through R-loop formation. Nat. Plants 3, 17053 (2017).

37. Zhang, M. et al. A peptide encoded by circular form of LINC-PINT suppresses oncogenic transcriptional elongation in glioblastoma. Nat. Commun. 9, 4475 (2018)

38. Orkin, S. H. \& Zon, L. I. Hematopoiesis: an evolving paradigm for stem cell biology. Cell 132, 631-644 (2008).

39. Nicolet, B. P. et al. Circular RNA expression in human hematopoietic cells is widespread and cell-type specific. Nucleic Acids Res. 46, 8168-8180 (2018).

40. Biswas, S. K. \& Mantovani, A. Macrophage plasticity and interaction with lymphocyte subsets: cancer as a paradigm. Nat. Immunol. 11, 889-896 (2010).

41. Zhang, Y., Zhang, Y., Li, X., Zhang, M. \& Lv, K. Microarray analysis of circular RNA expression patterns in polarized macrophages. Int. J. Mol. Med. 39 373-379 (2017).

42. Agirre, X. \& Meydan, C. Long non-coding RNAs discriminate the stages and gene regulatory states of human humoral immune response. Nat. Commun. 10, 821 (2019)

43. Deng, $T$. et al. Calcitonin generelated peptide induces IL6 expression in RAW264.7 macrophages mediated by mmu_circRNA_007893. Mol. Med. Rep. 16, 9367-9374 (2017).

44. Parish, S. T., Wu, J. E. \& Effros, R. B. Sustained CD28 expression delays multiple features of replicative senescence in human CD8 T lymphocytes. J. Clin Immunol. 30, 798-805 (2010).

45. Wang, Y. H., Yu, X. H., Luo, S. S. \& Han, H. Comprehensive circular RNA profiling reveals that circular RNA100783 is involved in chronic CD28associated CD8(+)T cell ageing. Immun. Ageing 12, 17 (2015).

46. Holdt, L. M et al. Circular non-coding RNA ANRIL modulates ribosomal RNA maturation and atherosclerosis in humans. Nat. Commun. 7, 12429 (2016).

47. Maass, P. G. et al. A map of human circular RNAs in clinically relevant tissues. J. Mol. Med. 95, 1179-1189 (2017).

48. Li, J. et al. Identification and characterization of a conservative $W$ chromosome-linked circRNA in half-smooth tongue sole (Cynoglossus 
semilaevis) reveal its female-biased expression in immune organs. Fish Shellfish Immunol. 82, 531-535 (2018).

49. Tagawa, T. et al. Discovery of Kaposi's sarcoma herpesvirus-encoded circular RNAs and a human antiviral circular. RNA 115, 12805-12810 (2018).

50. Zhang, X. et al. Identification and comparison of novel circular RNAs with associated co-expression and competing endogenous RNA networks in pulmonary tuberculosis. Oncotarget 8, 113571-113582 (2017).

51. Zhang, P. F. et al. Circular RNA circTRIM33-12 acts as the sponge of MicroRNA-191 to suppress hepatocellular carcinoma progression. Mol. Cancer 18, 105 (2019).

52. Weng, Q. et al. Global microarray profiling identified hsa_circ_0064428 as a potential immune-associated prognosis biomarker for hepatocellular carcinoma. J. Med. Genet. 56, 32-38 (2019).

53. Zhang, F. et al. Comprehensive analysis of circRNA expression pattern and circRNA-miRNA-mRNA network in the pathogenesis of atherosclerosis in rabbits. Aging 10, 2266-2283 (2018).

54. Zhang, Y. et al. GDF15 regulates Malat-1 circular RNA and inactivates NFkappaB signaling leading to immune tolerogenic DCs for preventing alloimmune rejection in heart. Transplant. Front. Immunol. 9, 2407 (2018).

55. Ma, X. et al. Differentially expressed non-coding RNAs induced by transmissible gastroenteritis virus potentially regulate inflammation and NFkappaB pathway in porcine intestinal epithelial cell line. BMC Genomics 19 747 (2018)

56. Zhang, Y. et al. Crosstalk in competing endogenous RNA networks reveals new circular RNAs involved in the pathogenesis of early HIV infection. J. Transl. Med. 16, 332 (2018).

57. Fu, Y., Wang, J., Qiao, J. \& Yi, Z. Signature of circular RNAs in peripheral blood mononuclear cells from patients with active tuberculosis. J. Cell Mol. Med. 23, 1917-1925 (2018).

58. Ng, W. L. et al. Inducible RasGEF1B circular RNA is a positive regulator of ICAM-1 in the TLR4/LPS pathway. RNA Biol. 13, 861-871 (2016).

59. Moreland, J. G., Fuhrman, R. M., Pruessner, J. A. \& Schwartz, D. A. CD11b and intercellular adhesion molecule-1 are involved in pulmonary neutrophil recruitment in lipopolysaccharide-induced airway disease. Am. J. Respir. Cell Mol. Biol. 27, 474-480 (2002).

60. Yang, M., Liu, J., Piao, C., Shao, J. \& Du, J. ICAM-1 suppresses tumor metastasis by inhibiting macrophage $\mathrm{M} 2$ polarization through blockade of efferocytosis. Cell Death Dis. 6, e1780 (2015)

61. Wu, Y., Zhang, Y., Zhang, Y. \& Wang, J. J. CircRNA hsa_circ_0005105 upregulates NAMPT expression and promotes chondrocyte extracellular matrix degradation by sponging miR-26a. Cell Biol. Int. 41, 1283-1289 (2017).

62. Liang, J. et al. Circular RNA expression profile analysis of severe acne by RNASeq and bioinformatics. J. Eur. Acad. Dermatol. Venereol. 32, 1986-1992 (2018).

63. Zhang, X. L., Xu, L. L. \& Wang, F. Hsa_circ_0020397 regulates colorectal cancer cell viability, apoptosis and invasion by promoting the expression of the miR138 targets TERT and PD-L1. Cell Biol. Int. 41, 1056-1064 (2017).

64. Kakavand, H. et al. PD-L1 expression and immune escape in melanoma resistance to MAPK inhibitors. Clin. Cancer Res. 23, 6054-6061 (2017).

65. Noguchi, T. et al. Temporally distinct PD-L1 expression by tumor and host cells contributes to immune escape. Cancer Immunol. Res. 5, 106-117 (2017).

66. Sharif, K, Amital, H. \& Shoenfeld, Y. The role of dietary sodium in autoimmune diseases: the salty truth. Autoimmun. Rev. 17, 1069-1073 (2018).

67. Zhang, Z. \& Zhang, R. Epigenetics in autoimmune diseases: pathogenesis and prospects for therapy. Autoimmun. Rev. 14, 854-863 (2015).

68. Watad, A. et al. Seasonality and autoimmune diseases: the contribution of the four seasons to the mosaic of autoimmunity. J. Autoimmun. 82, 13-30 (2017).

69. Mike, E. V. et al. Neuropsychiatric systemic lupus erythematosus is dependent on sphingosine-1-phosphate signaling. Front. Immunol. 9, 2189 (2018).

70. Fava, A. \& Petri, M. Systemic lupus erythematosus: diagnosis and clinical management. J. Autoimmun. 96, 1-13 (2019).

71. Li, L. J. et al. Circular RNA expression profile and potential function of hsa_circ_0045272 in systemic lupus erythematosus. Immunology $\mathbf{1 5 5}$ 137-149 (2018).

72. Zhang, C. et al. The down-regulation of hsa_circ_0012919, the sponge for miR-125a-3p, contributes to DNA methylation of CD11a and CD70 in CD4(+) T cells of systemic lupus erythematous. Clin. Sci. 132, 2285-2298 (2018).

73. Pan, Q. et al. Basophil recruitment to skin lesions of patients with systemic lupus erythematosus mediated by CCR1 and CCR2. Cell. Physiol. Biochem. $\mathbf{4 3}$ 832-839 (2017).
74. Kwon, S. J. et al. KLF13 cooperates with c-Maf to regulate IL-4 expression in CD4+T cells. J. Immunol 192, 5703-5709 (2014).

75. Wang, X., Zhang, C., Wu, Z., Chen, Y. \& Shi, W. CirclBTK inhibits DNA demethylation and activation of AKT signaling pathway via miR-29b in peripheral blood mononuclear cells in systemic lupus erythematosus. Arthr. Res. Ther. 20, 118 (2018).

76. Macintyre, A. N. et al. Protein kinase B controls transcriptional programs that direct cytotoxic $\mathrm{T}$ cell fate but is dispensable for $\mathrm{T}$ cell metabolism. Immunity 34, 224-236 (2011)

77. Zhang, C., Huang, J., Chen, Y. \& Shi, W. Low expression and clinical value of hsa_circ_0049224 and has_circ_0049220 in systemic lupus erythematous patients. Med. Sci. Monitor 24, 1930-1935 (2018).

78. Wei, Z. J., Liu, J. \& Qin, J. miR-138 suppressed the progression of osteoarthritis mainly through targeting p65. Eur. Rev. Med. Pharmacol. Sci. 21, 2177-2184 (2017).

79. Zhou, Z. B., Du, D., Huang, G. X., Chen, A. \& Zhu, L. Circular RNA Atp9b, a competing endogenous RNA, regulates the progression of osteoarthritis by targeting miR-138-5p. Gene. 646, 203-209 (2018).

80. Ouyang, Q. et al. Using plasma circRNA_002453 as a novel biomarker in the diagnosis of lupus nephritis. Mol. Immunol. 101, 531-538 (2018).

81. Mendez-Huergo, S. P. et al. Clinical relevance of Galectin-1 and Galectin-3 in rheumatoid arthritis patients: differential regulation and correlation with disease activity. Front. Immunol. 9, 3057 (2018).

82. de Moel, E. C. et al. In rheumatoid arthritis, changes in autoantibody levels reflect intensity of immunosuppression, not subsequent treatment response. Arthr. Res. Ther. 21, 28 (2019).

83. Zheng, F., Yu, X., Huang, J. \& Dai, Y. Circular RNA expression profiles of peripheral blood mononuclear cells in rheumatoid arthritis patients, based on microarray chip technology. Mol. Med. Rep. 16, 8029-8036 (2017).

84. Sheng, Y. J. et al. Follow-up study identifies two novel susceptibility loci PRKCB and 8p11.21 for systemic lupus erythematosus. Rheumatology (Oxford, England) 50, 682-688 (2011).

85. Tang, X. et al. Elevated expression of ciRS-7 in peripheral blood mononuclear cells from rheumatoid arthritis patients. Diagn. Pathol. 14, 11 (2019).

86. Mitra, A., Raychaudhuri, S. K. \& Raychaudhuri, S. P. IL-22 induced cell proliferation is regulated by PI3K/Akt/mTOR signaling cascade. Cytokine $\mathbf{6 0}$, 38-42 (2012).

87. Wu, X. et al. Gambogic acid suppresses inflammation in rheumatoid arthritis rats via PI3K/Akt/mTOR signaling pathway. Mol. Med. Rep. 16, 7112-7118 (2017).

88. Li, B. \& Li, N. Hsa_circ_0001859 regulates ATF2 expression by functioning as an MiR-204/211 sponge in human rheumatoid. Arthritis 2018, 9412387 (2018).

89. Ferrandiz, M. L., Nacher-Juan, J. \& Alcaraz, M. J. Nrf2 as a therapeutic target for rheumatic diseases. Biochem. Pharmacol. 152, 338-346 (2018).

90. Yang, J. H. et al. The differentially expressed circular RNAs in the Substantia Nigra and Corpus Striatum of Nrf2-knockout mice. Cell. Physiol. Biochem. 50, 936-951 (2018).

91. Hemmer, B., Kerschensteiner, M. \& Korn, T. Role of the innate and adaptive immune responses in the course of multiple sclerosis. Lancet. Neurol. $\mathbf{1 4}$ 406-419 (2015).

92. Palace, J. \& Robertson, N. Modifying disability in progressive multiple sclerosis. Lancet 383, 2189-2191 (2014).

93. Cardamone, G. et al. The Characterization of GSDMB splicing and backsplicing profiles identifies novel isoforms and a circular ma that are dysregulated in multiple sclerosis. Int. J. Mol. Sci. 18, pii: E576 (2017).

94. Shaker, O. G. \& Mahmoud, R. H. LncRNAs, MALAT1 and Inc-DC as potential biomarkers for multiple sclerosis diagnosis. Biosci. Rep. 39, pii: BSR20181335 (2019).

95. Cardamone, G. et al. Not only cancer: the long non-coding RNA MALAT1 affects the repertoire of alternatively spliced transcripts and circular RNAs in multiple sclerosis. Hum. Mol. Genet. 28, 1414-1428 (2018).

96. Greb, J. E. et al. Psoriasis. Nat. Rev. Dis. Primers 2, 16082 (2016).

97. Liu, R. et al. Mesenchymal stem cells in psoriatic lesions affect the skin microenvironment through circular RNA. Exp. Dermatol. 28, 292-299 (2019).

98. Seif, F. et al. The role of JAK-STAT signaling pathway and its regulators in the fate of T helper cells. Cell Commun. Signal. 15, 23 (2017).

99. Qiao, M. et al. Circular RNA expression profile and analysis of their potential function in psoriasis. Cell. Physiol. Biochem. 50, 15-27 (2018).

100. Liu, R. et al. Characterisation of the circular RNA landscape in mesenchymal stem cells from psoriatic skin lesions. Eur. J. Dermatol. 29, 29-38 (2019). 
101. Mousa, H. S. et al. Novel therapeutics for primary biliary cholangitis: toward a disease-stage-based approach. Autoimmun. Rev. 15, 870-876 (2016).

102. Zheng, J., Li, Z., Wang, T., Zhao, Y. \& Wang, Y. Microarray expression profile of circular RNAs in plasma from primary biliary cholangitispatients. Cell Physiol. Biochem. 44, 1271-1281 (2017).

103. Kang, G. J. et al. Novel involvement of miR-522-3p in high-mobility group box 1 -induced prostaglandin reductase 1 expression and reduction of phagocytosis. Biochim. Biophys. Acta. Mol. Cell Res. 1864, 625-633 (2017).

104. Hu, M. et al. Circular RNA expression profiles of persistent atrial fibrillation in patients with rheumatic heart disease. Anatol. J. Cardiol. 21, 2-10 (2019).

105. Miao, Q., Zhong, Z., Jiang, Z., Lin, Y. \& Ni, B. RNA-seq of circular RNAs identified circPTPN22 as a potential new activity indicator in systemic lupus erythematosus. Lupus 28, 520-528 (2019).

106. Zhang, M. Y. et al. Differentially expressed circular RNAs in systemic lupus erythematosus and their clinical significance. Biomed. Pharmacother. 107, 1720-1727 (2018).

107. Li, S. et al. Microarray expression profile of circular RNAs and mRNAs in children with systemic lupus erythematosus. Clin. Rheumatol. 38, 1339-1350 (2019)

108. Ouyang, Q. et al. Microarray expression profile of circular RNAs in peripheral blood mononuclear cells from rheumatoid arthritis patients. Cell. Physiol. Biochem. 42, 651-659 (2017).

109. Luo, Q. et al. Identification of circular RNAs hsa_circ_0044235 in peripheral blood as novel biomarkers for rheumatoid arthritis. Clin. Exp. limmunol. 194 118-124 (2018).
110. Iparraguirre, L. et al. Circular RNA profiling reveals that circular RNAs from ANXA2 can be used as new biomarkers for multiple sclerosis. Hum. Mol. Genet. 26, 3564-3572 (2017).

111. Weiss, R. et al. Cross-reactivity between annexin A2 and Beta-2-glycoprotein I in animal models of antiphospholipid syndrome. Immunol. Res. 65, 355-362 (2017).

112. Cheung, K. F. et al. Annexin I-binding immunoglobulins in patients with lupus nephritis and their correlation with disease manifestations. Clin. Sci. 131, 653-671 (2017)

113. Chen, Y. G. et al. Sensing self and foreign circular RNAs by intron identity. Mol. Cell 67, 228-238.e225 (2017).

114. Xia, P. et al. A circular RNA protects dormant hematopoietic stem cells from DNA sensor cGAS-mediated exhaustion. Immunity 48, 688-701.e687 (2018).

115. Yang, Y. G., Lindahl, T. \& Barnes, D. E. Trex1 exonuclease degrades ssDNA to prevent chronic checkpoint activation and autoimmune disease. Cell 131 873-886 (2007).

116. Thomas, C. A. et al. Modeling of TREX1-dependent autoimmune disease using human stem cells highlights L1 accumulation as a source of neuroinflammation. Cell Stem Cell 21, 319-331.e318 (2017).

117. Liu, C. X. et al. Structure and degradation of circular RNAs regulate PKR activation in innate immunity. Cell 177, 865-880.e821 (2019).

118. Cheng, J. et al. Downregulation of hsa circ 0068087 ameliorates TLR4/NFkappaB/NLRP3 inflammasome-mediated inflammation and endothelial cell dysfunction in high glucose conditioned by sponging miR-197. Gene. 709 1-7 (2019). 